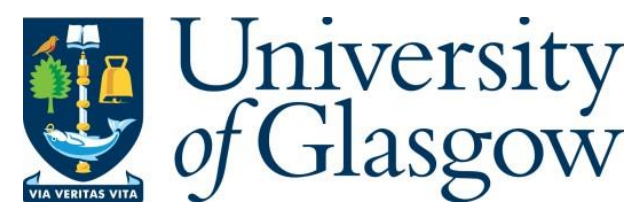

Gallacher, K., Millar, R. W., Griskevivuite, U., Baldassarre, L., Sorel, M., Ortolani, M. and Paul, D. J. (2019) TuA4.1 - Mid-infrared Sensing with Ge on Si Waveguides (Invited). In: 2019 IEEE Photonics Society Summer Topical Meeting Series (SUM), Ft. Lauderdale, FL, USA, 08-10 Jul 2019, ISBN 9781728105970.

There may be differences between this version and the published version. You are advised to consult the publisher's version if you wish to cite from it.

http://eprints.gla.ac.uk/193484/

Deposited on: 20 August 2019

Enlighten - Research publications by members of the University of Glasgow http://eprints.gla.ac.uk 


\title{
Mid-infrared Sensing with Ge on Si Waveguides
}

\author{
Kevin Gallacher*, Ross W. Millar*, Ugnė Griškevičiūtė*, Leonetta Baldassarre ${ }^{\dagger}$, \\ Marc Sorel $^{*}$ Michele Ortolani $^{\dagger}$ and Douglas J. Paul ${ }^{*}$ \\ ${ }^{*}$ School of Engineering, University of Glasgow, Rankine Building, Oakfield Avenue, Glasgow G12 8LT, U.K. \\ $\dagger$ Dipartimento di Fisica, Sapienza Universitá di Roma, Piazzale Aldo Moro 5, I-00185 Rome, Italy
}

\begin{abstract}
Ge-on-Si waveguides with losses below $5 \mathrm{~dB} / \mathrm{cm}$ across 7.5 to $11 \mu \mathrm{m}$ wavelength are demonstrated. Sidewall etch roughness was measured using atomic force microscopy to investigate the waveguide loss mechanisms. Mid-infrared spectroscopy of poly(methyl methacrylate) was demonstrated using the Ge waveguides for mid-infrared sensing.
\end{abstract}

The mid-infrared (MIR) part of the electromagnetic spectrum provides the strongest molecular absorption signatures for molecules to be identified [1]. Simple gas molecules such as $\mathrm{CO}_{2}, \mathrm{CO}$ or $\mathrm{NO}_{2}$ have strong absorption lines in the 3 to $5 \mu \mathrm{m}$ atmospheric transparent window but many larger molecules have unique signatures in the 6 to $20 \mu \mathrm{m}$ fingerprint region [1]. The 8 to $14 \mu \mathrm{m}$ wavelengths are extremely important as this is a second transparent atmospheric window where molecular absorption and vibrational lines allow unique identification of many chemical and biological molecules with potential lab-on-a-chip applications for environmental monitoring, healthcare and security applications [2].

The gold standard for MIR identification is the Fourier Transform InfraRed (FTIR) spectrometer, however, such systems require expert users and are expensive. Recent research has been investigating lab-on-a-chip based approaches using semiconductor waveguides with the potential to significantly reduce the cost for molecular identification by non-experts [3]. $\mathrm{Si}$ photonics platforms have low $\mathrm{Si}$ material losses below 10 $\mu \mathrm{m}$ but the losses in $\mathrm{SiO}_{2}$ and $\mathrm{Si}_{3} \mathrm{~N}_{4}$ become significant at 4 and $6.7 \mu \mathrm{m}$ respectively [3] precluding the use of the 8 to14 $\mu \mathrm{m}$ atmospheric window and many of the unique explosive and chemical weapons lines between 10 and $14.5 \mu \mathrm{m}$ [4].

We have previously demonstrated Ge-on-Si low loss waveguides [5], plasmonic antenna [2], intersubband photodetectors [6] and third harmonic generation [7] using the Ge-on-Si platform in the 8 to $14 \mu \mathrm{m}$ wavelength mid-infrared region. Here we demonstrate a sidewall dependent scattering mechanism which effects the losses of the waveguides. An atomic force microscope (AFM) was used to measure the sidewall roughness for different plasma etches and we demonstrate how the waveguide losses are increased as the root mean squared (rms) amplitude of the roughness increases. We then demonstrate molecular absorption and vibration sensing using the $\mathrm{Ge}$ waveguides covered with poly(methyl methacrylate) (PMMA) and compare the transmission results to FTIR measurements.

The $2 \mu \mathrm{m}$ thick Ge epilayers were grown on $150 \mathrm{~mm} \mathrm{Si}$ (001) wafers using an ASM Epsilon 2000 chemical vapor deposition tool. Electron beam lithography and fluorine based dry etches were used to pattern $4 \mu \mathrm{m}$ wide and $1 \mu \mathrm{m}$ deep rib
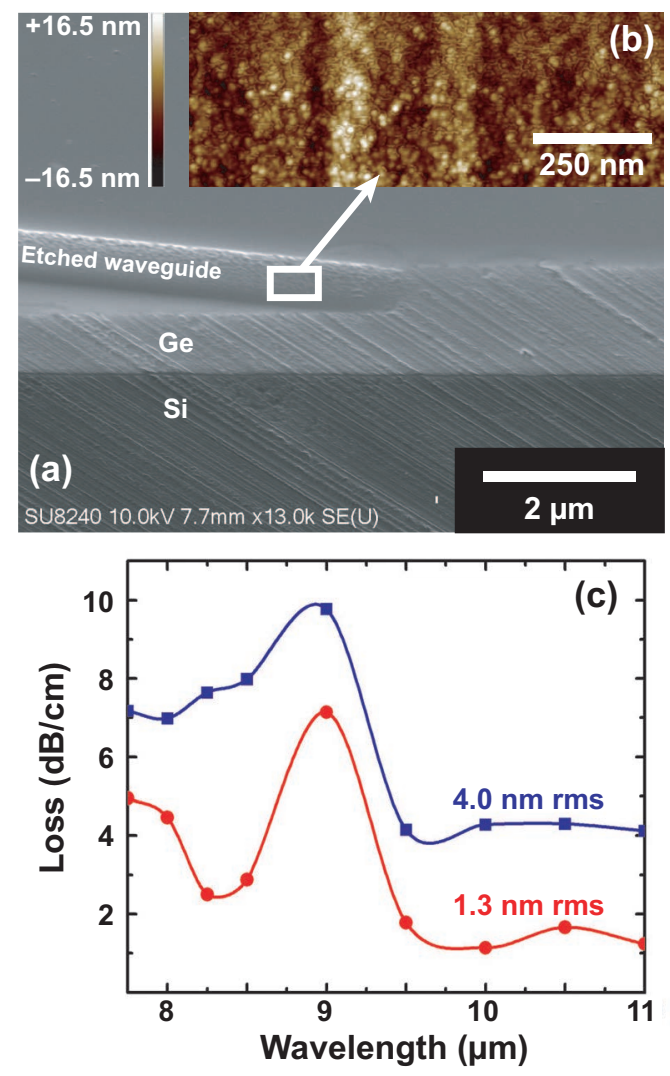

Fig. 1. (a) A SEM image of a Ge-on-Si rib waveguide which has been sawn and polished back to allow AFM measurements of the sidewall roughness. (b) The AFM measurement of the sidewall. (c) The waveguide losses versus wavelength from 2 different etches that have different sidewall roughnesses.

waveguides. The Fabry-Perot technique was used to measure waveguide losses using a 7.5 to $11.5 \mu \mathrm{m}$ wavelength quantum cascade laser and a DLaTGS detector [5]. Once losses were measured for samples etched using different fluorine etch recipes, the sampled were polished back at an angle to the waveguide to allow a carbon nanotube AFM tip on a Bruker Dimension Icon AFM to measure the roughness (see Fig. 1(a)). The insert to Fig. 1(a) presents the AFM image of the Ge sidewall with an extracted roughness amplitude of $4.0 \mathrm{~nm} \mathrm{rms}$ whilst a second etch process (not shown) was measured with an amplitude of $1.3 \mathrm{~nm}$ rms.

Figure 1(b) presents the losses as measured by the FabryPerot technique [5] for two different roughness amplitudes. The large peak around $9 \mu \mathrm{m}$ wavelength is related to the 
absorption from interstitial oxygen from the $\mathrm{Cz} \mathrm{Si}$ substrate. If this $\mathrm{Si}-\mathrm{O}$ absorption is removed then the losses are below $5 \mathrm{~dB} / \mathrm{cm}$ across the measured range for the sample with a roughness amplitude of $1.3 \mathrm{~nm} \mathrm{rms}$. Above $9.5 \mu \mathrm{m}$ wavelength, the losses are reduced to $\sim 1 \mathrm{~dB} / \mathrm{cm}$. The effect of higher roughness in increasing the waveguide losses across the complete measurement range is demonstrated by the higher loss in the sample with measured $4.0 \mathrm{~nm} \mathrm{rms}$ roughness amplitudes but such amplitudes in commonly used analytical scattering models should cause negligible loss [8], [5].

To demonstrate molecular absorption and vibration sensing using the Ge on Si waveguides, PMMA was spin coated onto the waveguides and the transmission was measured (see Fig. 2). An oxygen plasma was used to remove the PMMA leaving only a $1 \mathrm{~mm}$ long strip over the waveguides. The results in Fig. 2 are compared to transmission results from FTIR transmission measurements taken in a Bruker 66vs FTIR in step-scan mode. The C-O-C vibration stretching modes [9] are clearly visible at $8.39 \mu \mathrm{m}$ and the skeletal C-C vibration at $9.38 \mu \mathrm{m}$. The larger absorption from the waveguide samples is related to the longer effective propagation length through the PMMA analyte from the evanescent coupling of the mode with the analyte. Despite the relatively low modal overlap with the evanescent coupling, the $\sim 1 \mathrm{~mm}$ propagation length compared to $\sim 40 \mathrm{~nm}$ in the FTIR vertical transmission geometry provides a factor of $\geq 40$ higher absorption. This potential provides significant advantages for a wide range of applications including healthcare, security and environmental sensing.

We have experimentally measured the level of sidewall roughness on Ge-on-Si rib waveguides using AFM measurements and demonstrated a clear sidewall dependence of waveguide losses. Scattering using standard roughness loss models should be negligible for the roughness levels measured. Alternative sidewall dependent mechanisms are currently under investigation. Losses around $1 \mathrm{~dB} / \mathrm{cm}$ are demonstrated above $9.5 \mu \mathrm{m}$ wavelength for sidewall roughness of $1.3 \mathrm{~nm}$ rms. These low loss Ge-on-Si waveguides were used to demonstrate spectroscopy of PMMA with $\mathrm{C}-\mathrm{O}-\mathrm{C}$ and $\mathrm{C}-\mathrm{C}$ vibrational absorptions clearly visible. These results demonstrate that the Ge-on-Si platform has significant potential to produce lab-ona-chip sensors for mid-infrared applications.

\section{ACKNOWLEDGMENT}

The research was funded by U.K. EPSRC (EP/N003225/1).

\section{REFERENCES}

[1] J. M. Hollas, Modern Spectroscopy, 4th ed. Wiley, 2004.

[2] L. Baldassarre, E. Sakat, J. Frigerio, A. Samarelli, K. Gallacher, E. Calandrini, G. Isella, D. J. Paul, M. Ortolani, and P. Biagioni, "Midinfrared plasmon-enhanced spectroscopy with Ge antennas on $\mathrm{Si}$ substrates," Nano Lett., vol. 15, no. 11, pp. 7225-7231, 2015. [Online]. Available: http://dx.doi.org/10.1021/acs.nanolett.5b03247

[3] R. Soref, "Mid-infrared photonics in $\mathrm{Si}$ and Ge," Nat. Photon., vol. 4, no. 8, pp. 495-497, Aug 2010. [Online]. Available: http://dx.doi.org/10.1038/nphoton.2010.171

[4] E. R. Deutsch, P. Kotidis, N. Zhu, A. K. Goyal, J. Ye, A.Mazurenko, M. Norman, K. Zafiriou, M. Baier, and R. Connors, "Active and passive infrared spectroscopy for the detection of environmental threats," Proc. SPIE, vol. 9106, p. 9106, 2014. [Online]. Available: https://dx.doi.org/10.1117/12.2058544
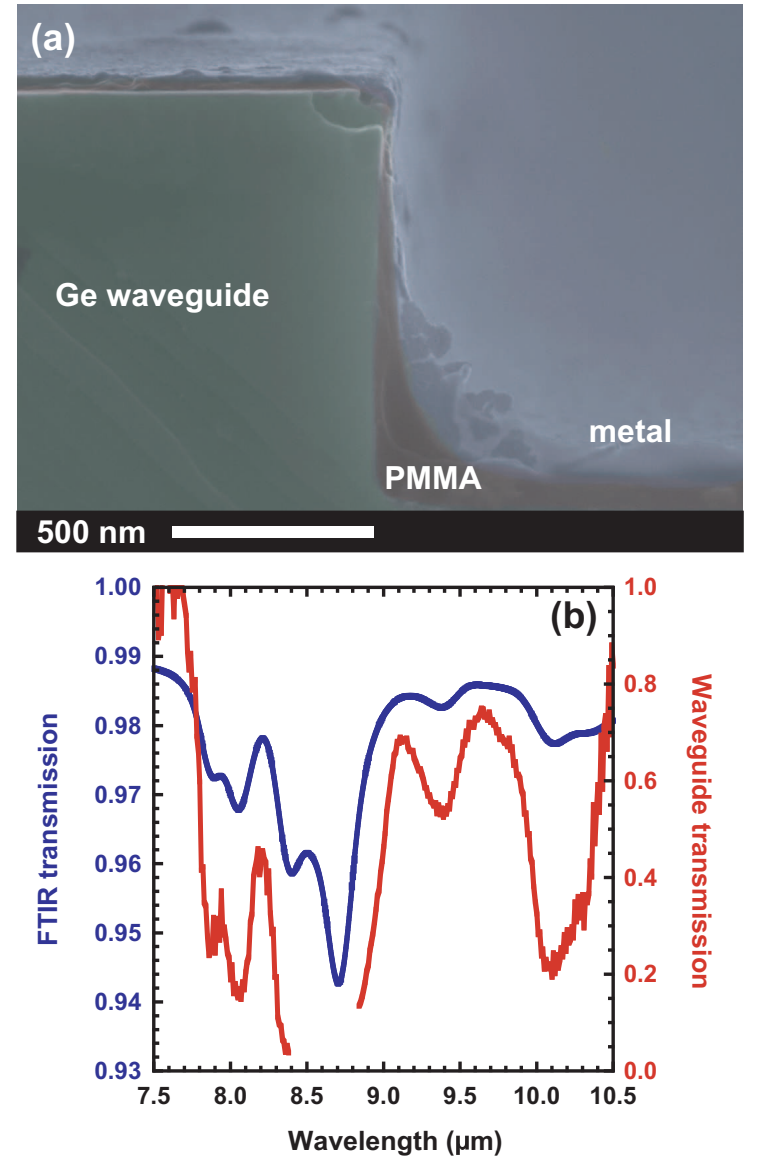

Fig. 2. (a) A SEM image of a Ge-on-Si rib waveguide with a thin layer of PMMA spun on top to undertake spectroscopy. The metal on top was sputtered coated before the SEM image was taken to reduce charge build up in the SEM and allow the thickness of the PMMA to be determined. (b) The FTIR transmission spectra of PMMA in blue compared to the transmission spectra of the waveguide coated with PMMA.

[5] K. Gallacher, R. Millar, U. Griškevičiūte, L. Baldassarre, M. Sorel, M. Ortolani, and D. J. Paul, "Low loss Ge-on-Si waveguides operating in the $8-14 \mu \mathrm{m}$ atmospheric transmission window," Opt. Exp., vol. 26, no. 20, pp. 25667-25675, Oct 2018. [Online]. Available: https://dx.doi.org/10.1364/OE.26.025667

[6] K. Gallacher, A. Ballabio, R. W. Millar, J. Frigerio, A. Bashir, I. MacLaren, G. Isella, M. Ortolani, and D. J. Paul, "Mid-infrared intersubband absorption from p-Ge quantum wells grown on $\mathrm{Si}$ substrates," Appl. Phys. Lett., vol. 108, no. 9, 2016. [Online]. Available: http://dx.doi.org/10.1063/1.4943145

[7] M. P. Fischer, A. Riede, K. Gallacher, J. Frigerio, G. Pellegrini, M. Ortolani, D. J. Paul, G. Isella, A. Leitenstorfer, P. Biagioni, and D. Brida, "Plasmonic mid-infrared third harmonic generation in germanium nanoantennas," Light: Sci. \& Apps., vol. 7, no. 1, p. 106, 2018. [Online]. Available: https://doi.org/10.1038/s41377-018-0108-8

[8] F. P. Payne and J. P. R. Lacey, "A theoretical analysis of scattering loss from planar optical waveguides," Opt. Quant. Elec., vol. 26, no. 10, pp. 977-986, Oct 1994. [Online]. Available: https://doi.org/10.1007/BF00708339

[9] A. Dazzi, A. Deniset-Besseau, and P. Lasch, "Minimising contributions from scattering in infrared spectra by means of an integrating sphere," Analyst, vol. 138, pp. 4191-4201, 2013. [Online]. Available: http://dx.doi.org/10.1039/C3AN00381G 